\section{Desafíos de la Educación Alimentaria Nutricional: construcción de espacios y vínculos en el nivel inicial}

\author{
Sandra Ravelli \\ Docente de la Facultad de Bioquímica \\ y Ciencias Biológicas. Universidad \\ Nacional del Litoral, Argentina. \\ sravell@fbcb.unl.edu.ar
}

\section{Adriana Panicia}

Docente del Jardín Maternal de la

Universidad Tecnológica Nacional -

Regional Santa Fe, Argentina.

direjm@frsf.utn.edu.ar

\author{
Érica Figueroa \\ Subsecretaria de Educación. \\ Municipalidad de Santa Fe, Argentina. \\ educacion@santafeciudad.gov.ar
}

Integración de la docencia y la extensión /

Intervenciones

RECEPCIÓN: 24/06/16

ACEPTACIÓN FINAL: 10/10/16

\section{Resumen}

Este artículo describe las intervenciones que se realizaron en el marco de un Proyecto de Extensión en Educación Experiencial de la Universidad Nacional del Litoral. La misma convocó a los alumnos avanzados que realizan las prácticas profesionales de la Licenciatura en Nutrición de la Facultad de Bioquímica y Ciencias Biológicas. El proyecto tiene por objeto la promoción de una alimentación saludable a través de propuestas didácticas y lúdicas e involucra diferentes ejes: sobre alimentación saludable e higiene de manos y de los alimentos en el nivel inicial. El artículo abordará las tres etapas que insumió el proyecto: capacitación y vinculación de los agentes (equipo extensionista, docentes, no docentes, alumnos de prácticas profesionales y familias), construcción de recursos didácticos con contenidos de nutrición y paralelamente una etapa de interacción e implementación de las actividades con la comunidad. Asimismo, se dará cuenta de los logros y dificultades que implicó su desarrollo.

\section{Palabras-clave}

- Nivel inicial

- Educación Alimentaria Nutricional

- Integración docencia-extensión

\section{Resumo}

Este artigo descreve as intervenções que se realizaram no âmbito do Projeto de Extensão em Educação Experiencial da "Universidad Nacional del Litoral". Ela convocou os estudantes avançados que realizam seus estágios profissionalizantes do curso de graduação de Nutrição na Faculdade de Bioquímica e Ciências Biológicas. O objetivo do projeto é promover a alimentação saudável através de propostas educativas e lúdicas, e envolve diferentes eixos: sobre alimentação saudável e higiene de mãos, e alimentos no nível inicial. O artigo abordará as três etapas que levou o projeto: capacitação e vinculação do pessoal (equipe de extensão, docentes e não-docentes, alunos do estágio profissionalizante, e famílias); construção de recursos didáticos com conteúdo nutricional; e paralelamente uma etapa de interação e implementação de atividades com a comunidade. Ademais serão informadas as conquistas e as dificuldades que originou o seu desenvolvimento.

Palavras-chave

- Nível Inicial

- Educação Alimentar e Nutricional

- Integração docência-extensão
Para citación de este artículo

Ravelli, S.; Panicia, A. y Figueroa, E. (2016). Desafíos de la Educación Alimentaria Nutricional: Construcción de espacios y vínculos en el nivel inicial. En Revista $+E$, versión digital (6), pp. 256-263. Santa Fe, Argentina: Ediciones UNL. 


\section{Introducción}

Este trabajo presenta las Propuestas de Extensión en Educación Experiencial (PEEE) en el nivel inicial para la promoción de una alimentación sana y segura realizadas por alumnos de las prácticas profesionales de la Licenciatura en Nutrición de la Facultad de Bioquímica y Ciencias Biológicas (FBCB) de la Universidad Nacional del Litoral (UNL).

Se destacan, en primer lugar, algunos antecedentes sobre proyectos educativos relacionados con salud, nutrición y alimentación en este grupo etáreo y se menciona la PEEE llevada adelante en 2014, la que al momento continúa evidenciando, que son muchas las propuestas y actividades programadas para niños que asisten a la escuela primaria y secundaria pero aún son escasas las destinadas al nivel inicial y preescolar.

Luego se fundamenta la importancia de la Educación Alimentaria Nutricional (EAN) y cómo se complementa con el juego para la utilización de estrategias educativas apropiadas a la edad.

Se describe además la relación entre las funciones de docencia, extensión e investigación que se pone de manifiesto en la propuesta y la proyección de las intervenciones en otros espacios e instituciones.

Las estrategias utilizadas para cumplir con los objetivos planteados describen las intervenciones, actividades y los juegos desarrollados sobre la base de los ejes y contenidos trabajados con los niños y comunidad en general, así como la transferencia de lo realizado y la potenciación de diversos lenguajes comunicacionales artísticos expresivos.

Hacia el final de este artículo, presentamos los logros y debilidades de la propuesta con la intención de mejorar y analizar de manera crítica aquellos obstáculos que fueron detectados en forma conjunta por el equipo extensionista y ponemos en claro los aspectos a mejorar.

Igualmente, se consideran las perspectivas y la importancia del proyecto en la formación del equipo extensionista que sincroniza y complementa las herramientas teóricas brindadas en el aula con espacios reales de vinculación para promover, en este caso, la salud y el desarrollo comunitario.

\section{Antecedentes}

En un trabajo compartido con el Instituto de Nutrición y Tecnología de los Alimentos (INTA) y el Ministerio de Educación de Chile, se evaluó que los niños tenían un bajo consumo de productos lácteos, verduras, frutas y pescado, y un alto consumo de productos elaborados ricos en grasas, azúcar y sal. Se incorporaron así al proyecto estrategias educativas que produjeran materiales educativos, como libros de educación en alimentación y nutrición para la enseñanza básica, guías para profesores y la para alumnos de enseñanza básica.
En otro estudio realizado en el mismo país, donde se evaluó una intervención en educación nutricional y actividad física en niños de 4 a 7 años, y donde uno de los ejes fue la capacitación de profesores para que apliquen un programa de educación nutricional, se obtuvo como resultado el incremento de conocimientos en cuanto a alimentación (Kain y otros, 2012). En la ciudad de Córdoba se realizaron cinco talleres educativos y dos tutorías con los docentes sobre alimentación saludable y su inclusión en la propuesta didáctica como contenidos transversales y se valoró el impacto positivamente, ya que los docentes diseñaron un proyecto sobre alimentación y nutrición que forma parte del Proyecto Educativo Institucional de la escuela y que involucra también al quiosco escolar, la población de padres de los escolares y el material didáctico diseñado y validado para dicha población, lo cual contribuye a mejorar la calidad de la intervención para promover hábitos alimentarios saludables y duraderos.

Para la intervención educativa se recurrió al Modelo Comunitario sustentado en metodologías constructivistas (Del Campo, 2010). Los antecedentes anteriores motivaron una propuesta de PEEE de la cátedra Práctica Profesional de la Licenciatura en Nutrición en el año 2014.

Como expresa Camilloni (2011), la Educación Experiencial es una estrategia de enseñanza con enfoque holístico que está destinada a relacionar el aprendizaje con la vida real. En este sentido, la posibilidad de participar en la experiencia impactó no solo en la formación integral de los alumnos ya que estrecharon lazos, compartieron experiencias, historias y datos del seno mismo de la comunidad, sino también en la tarea docente al jerarquizarla, en el currículo y en acciones pedagógicas que lograron resignificar la propia práctica profesional, la práctica docente y la práctica social. A partir de esta mirada se valoró interdisciplinariamente y se concluyó que son necesarias las prácticas de extensión en la asignatura con la incorporación de la educación alimentaria nutricional en nivel inicial.

El desafío de esta propuesta fue transmitir, implementar e intercambiar en este nivel contenidos relacionados con la alimentación y nutrición saludable, preparar un entorno lúdico favorable con actividades y recursos educativos que potencien el desarrollo infantil y se que integren el saber con el actuar, con el sentir y con el crear. Participaron docentes y coordinadores de nivel inicial, alumnos de la carrera de Licenciatura en Nutrición, especialistas en nutrición y docentes de artes gráficas y expresivas. Se realizó además un trabajo multisectorial que vinculó la Facultad de Bioquímica y Ciencias Biológicas con jardines de instituciones universitarias (Universidad Tecnológica Nacional), jardines municipales y la Subsecretaría de Educación de la Municipalidad de Santa Fe. Este trabajo interdisciplinario contribuyó a un diagnóstico situacional de cada jardín relevado, por lo que las 
actividades, propuestas y resoluciones a la problemática fueron diferentes en cada lugar de intervención educativa. Y, como se mencionó anteriormente, aportó a la valoración de la necesidad de acompañar en este nivel con prácticas de extensión destinadas a la EAN.

\section{Educación Alimentaria Nutricional en el nivel inicial}

La EAN se concibe como un proceso dinámico a través del cual los individuos, las familias y su comunidad adquieren, reafirman o cambian sus conocimientos, actitudes, habilidades y prácticas, actuando racionalmente en la producción, selección, adquisición, conservación, preparación y consumos de los alimentos, de acuerdo a sus pautas culturales, necesidades individuales y a la disponibilidad de recursos en cada lugar. (Ministerio de Educación, 2011). Debe orientarse a potenciar o modificar los hábitos alimentarios, involucrando a todos los miembros de la comunidad educativa; niños, padres, maestros y directivos; esto implica: descubrir y erradicar creencias, mitos y conductas erróneas; promoviendo una mayor consciencia sobre las múltiples funciones o roles que juega o debe jugar la alimentación en las diversas esferas de la vida, la salud, los aprendizajes, la producción, distribución y consumo de alimentos; y el énfasis que la educación debe asumir, sobre todo en la infancia, en el fomento de conceptos, actitudes y conductas claras y fundamentales sobre la alimentación (De La Cruz, 2014). En este sentido, se debe poner énfasis, especialmente en el nivel educativo Inicial, y en todos los actores que en ella actúan; niños, maestros, la familia y la comunidad en general.

Esta propuesta atraviesa a la comunidad educativa, debido a que los docentes participaron en las capacitaciones previas, y la familia junto a los niños en forma activa compartiendo las actividades, juegos, efemérides nutricionales, jornadas especiales, lecturas relacionadas, carteleras saludables entre otras.

\section{El juego y la EAN}

El juego es la expresión de estados intencionales, las representaciones en la conciencia construidas a partir de lo que el niño sabe y sobre lo que está aprendiendo de eventos que suceden. Consiste en actividades espontáneas, que ocurren naturalmente con objetos que comprometen la atención y el interés del niño (Lifter, 2000).

Esta definición coloca especial énfasis en el compromiso del niño con la actividad lúdica. Estudios recientes dan soporte empírico a la relación entre este compromiso activo y el aprendizaje del niño. Así, los niños ponen mayor atención a las actividades evolutivamente nuevas que a las que les son relativamente bien conocidas. En consecuencia, las actividades evolutivamente relevantes son definidas como "actividades que representan nuevos aprendizajes", están ubicadas en el umbral del aprendizaje, sobre las cuales el niño pone atención en el esfuerzo de interpretar y dar sentido a los eventos que van sucediendo.

Ortega Ruiz (1992) expuso que la fuerza motivadora y el interés intrínseco que los niños incluyen en sus juegos nacen de la propia naturaleza epistemológica del ser humano; por eso juego y aprendizaje necesariamente están relacionados. El problema es cómo hacer un uso educativo de esta fuente natural de conocimiento y desarrollo. Durante el juego, los niños expresan sus propias ideas sobre los asuntos que éste implica y, de esta forma, manifiestan sus esquemas conceptuales y los someten al juicio y aprobación de los compañeros, que rectifican, negociadamente, aquello que no es correcto, no es útil o de lo que hay un concepto mejor. De esta manera, el juego puede ser considerado un escenario pedagógico natural que permite al profesor, si conoce bien a los jugadores, establecer estrategias de aprendizaje basadas en él.

La observación de los juegos cotidianos, tradicionales, juegos trabajo, juegos libres, fue clave a la hora de la elaboración de material específico y acompañó en su totalidad el proceso educativo. El material desarrollado implicó, ajustes propios, resoluciones interdisciplinarias, y procesos de validaciones. Los juegos pudieron ser utilizados tanto dentro del horario de actividades formales en las salas como fuera de ellas. En la construcción de este tipo de recursos primaron los que abordaban tres grandes ejes: Higiene de los alimentos y lavado de manos, Conociendo y preparando nuestros alimentos y Nuestra huerta. Así se utilizaron: juegos de locomoción y traslado, juegos de mesa, actividades que estimulaban la oralidad como adivinanzas y rimas, juegos de coordinación, gráficos y audiovisuales y recursos audiovisuales y dramatizaciones. El juego en esta etapa constituyó un instrumento cotidiano con el que el niño captó su entorno y experimentó en él. Así, se buscó brindar EAN mediante la construcción y sistematización de aquellos instrumentos lúdicos y artísticos que se validaron en conjunto con los alumnos de las prácticas profesionales, docentes del nivel, familiares y niños.

\section{Actividades de extensión, docencia e investigación} Como parte del acercamiento a los distintos jardines y barrios donde estas instituciones se enclavan, el trabajo de extensión comenzó con el relevamiento de datos referidos a la situación sociodemográfica, a la organización de las diversas instituciones en cuanto a hábitos de higiene, aseo, alimentación, colaciones, existencia de espacios de trabajo, de juego, tipo de material existente, así como docentes y no docentes interesados en llevar adelante el proyecto. En ese relevamiento se detectaron debilidades y problemáticas, fortalezas y posibilidades para la resolución de problemas y el aporte de ideas propias e inherentes a cada jardín. 


\section{6}

la valoración de la práctica de extensión fue

diferente para los alumnos que participaron

en ella y para los que no lo hicieron

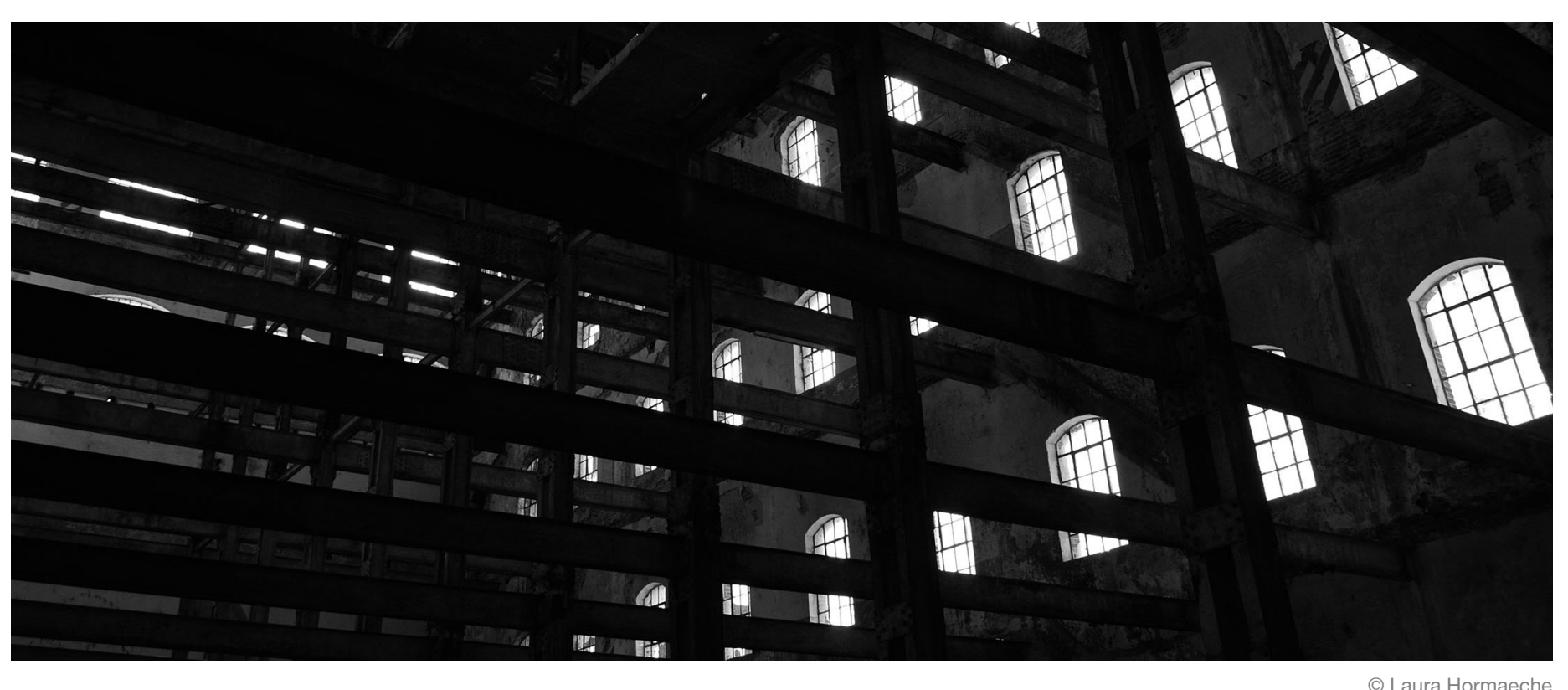

Al inicio de la PEEE, enmarcada en una perspectiva integral que propone la adquisición de conocimientos disciplinares relacionados con las competencias laborales vinculados al niño, la alimentación y su entorno, y además la internalización de valores, actitudes y conductas que los acerquen al medio y la comunidad, se convocó a ocho alumnos interesados en el nivel inicial, acompañados por docentes tutores de práctica de la carrera de Nutrición, además de docentes voluntarias de otros espacios curriculares, coordinadora del nivel inicial de los jardines municipales y privados y sus docentes. Recibieron capacitación correspondiente a través de seminarios y cursos de formación en los diferentes jardines y en la FBCB, los que sumaron aproximadamente 36 horas. La evaluación de los mismos fue teórica y práctica y el objetivo fue generar en grupos juegos y recursos didácticos basados en los ejes propuestos. Los trabajos fueron valorados por los integrantes de las capacitaciones y seminarios. La socialización de las propuestas fue realizada tanto in situ como en las redes sociales (grupos de Facebook, entorno virtual), enriqueció la realización del proyecto y posibilitó a los estudiantes aplicar los conocimientos adquiridos al vincular el aprendizaje en el aula con la extensión en su formación universitaria.
Como todo proceso educativo, hubo obstáculos en la realización de las intervenciones, las que se procuró subsanar a través de reuniones individuales, grupales, y foros de discusión donde se acordaron instancias de solución y acercamiento al problema en cuestión. Los problemas presentados fueron de diferente génesis: administrativos, pedagógicos, culturales y de relaciones humanas, entre otros. Los docentes de la cátedra y de las instituciones orientaron a través de la repregunta y reflexión sobre las problemáticas y discusiones llevadas adelante en los diferentes espacios.

Cabe destacar que la valoración de la práctica de extensión fue diferente para los alumnos que participaron en ella y para los que no lo hicieron, eso se evidenció en las apreciaciones al momento de socializar lo vivido. Los participantes en la PEEE resaltaron la aproximación a los niños y familias, la construcción de recursos didácticos para interactuar y compartir los mensajes de alimentación saludable y la búsqueda de actividades y contenidos acordes a la edad. Fue un trabajo arduo pero enriquecedor, que evidenció el compromiso y la actitud profesional de los extensionistas. La función docente fue de utilidad para intercambiar experiencias y contenidos relacionados con la alimentación, nutrición e higiene de 
alimentos para la comunidad educativa de los jardines maternales así como para el acompañamiento y preparación de los alumnos de las prácticas profesionales en donde desarrollan uno de sus roles esenciales a la hora de adquirir competencias profesionales: educación y promoción de la salud.

Con respecto a la investigación, la propuesta no fue sistematizada. Si bien la observación participante, la recopilación de datos, la evaluación y devolución de los involucrados en el proyecto fue continua y escrita en informes compartidos entre las diferentes instituciones, es actualmente cuando se profundiza la misma a través de la realización de trabajos finales por parte de cuatro alumnas para la obtención del título de Licenciado en Nutrición y lo que podrá ser utilizado como plataforma de futuros proyectos de extensión, docencia e investigación.

\section{Estrategias para cumplir con los objetivos planteados}

Ante la demanda de docentes y padres en diferentes encuentros e intervenciones aisladas realizados con anterioridad, se proyectaron encuentros intercátedras: Práctica Profesional, Alimentación Infantil y Educación para la Salud y Economía Familiar, para generar propuestas e ideas para la construcción de entornos más saludables y consensuar contenidos relacionados con alimentación, actividad física y recreación. Se proyectó una PEEE en la que los alumnos de $5^{\circ}$ año de la Licenciatura en Nutrición acompañaron durante 2014 las siguientes propuestas:

- Encuentros de capacitación y diagnóstico con docentes del nivel inicial que se realizaron al comienzo del proyecto, con implementación de estrategias participativas y de carácter teórico-práctico. Los ejes que se abordaron fueron: higiene de los alimentos y aseo personal y conocimiento y producción de alimentos. Éstos fueron planteados en unidades didácticas (generalmente trimestrales), se presentaron recursos didácticos innovadores, como juegos ${ }^{1}$ con materiales coloridos, libros de cuentos, obras infantiles, canciones, adivinanzas y rimas y se interactuó con profesionales de distintas disciplinas como música, diseño gráfico, teatro, expresión corporal. Se realizaron talleres de cocina que rescataron sabores y recetas familiares, se construyó un recetario institucional, se programaron fiestas y jornadas de carácter familiar en donde se socializaron las propuestas y se construyó la Cartelera Nutritiva.

- Diseño de diferentes espacios especialmente preparados para el desarrollo de contenidos de nutrición e higiene de los alimentos, tales como verdulería, supermercado, cocina familiar, en donde los niños simbolizaron actividades de la vida cotidiana mediante la utilización, observación, investigación, lavado, reconocimiento de colores, texturas, olores y sabores de alimentos y productos comunes, con alimentos conocidos y algunos no habituales en su alimentación.

- Promoción de la salud. Aprovechando los días o efemérides de salud, tales como el Día del Agua, Día Mundial de la Alimentación, Día de la Patria, los niños prepararon folletería, souvenirs, recetas para la comunidad sobre la temática alusiva.

- Se realizaron huertas con hierbas aromáticas y germinadores cuyo seguimiento permitió la observación del crecimiento de la planta hasta la preparación de alimentos con los productos cultivados. Además se degustaron verduras y preparaciones, como choclos, tortas y budines de diferentes hortalizas en una jornada con abuelos y familiares. Cada intervención quedó registrada a través de una tarjeta, folleto o recuerdo en los cuadernos de comunicaciones con el propósito de que llegue lo realizado en las diferentes salas a la familia y de transferir lo realizado y aprendido.

- Realización de una obra de teatro infantil. Como cierre del ciclo lectivo 2014, y para destacar el carácter interdisciplinario de la PEEE, se puso en escena una obra infantil donde participaron 12 nutri-actores estudiantes universitarios. La obra fue íntegramente desarrollada por estos estudiantes: desde su guión, actuación, vestuario, escenografía, sonido, imágenes, edición, y fue representada en seis oportunidades para jardines maternales, Centros de Acción Familiar y centros de día, a las que concurrieron 1200 niños y adultos de distintos puntos de la ciudad. Las actividades de índole artístico-expresiva y encuentros con la comunidad educativa se difundieron a través de medios oficiales de las instituciones de la ciudad y de las redes sociales. Se logró un acercamiento masivo de diferentes grupos, personas y entidades que siguieron aportando y sumándose a la propuesta.

Las producciones fueron presentadas en diferentes eventos: encuentros de cátedra, Congreso " 5 al día", "XII Congreso de Graduados en Nutrición", capacitaciones docentes, jornadas con diferentes jardines y artículos en diarios de la ciudad.

Se programó y se llevó adelante, debido al entusiasmo demostrado en el proyecto, un curso, con el Ministerio de Educación de la Nación, para docentes y promotores de salud interesados en nivel inicial como parte del Componente 2 de formación permanente.
1) Algunos de estos juegos fueron Dominó Saludable, Cocineritos, Cocina Mágica, Caja de sorpresas, rayuela
Nutritiva, Memo test, Juegos de tracción y transporte (con componentes nutricionales). 


\section{6}

con referencia a la asignatura, se

observó un crecimiento en el trabajo

interdisciplinar, la vinculación con

las instituciones y el medio

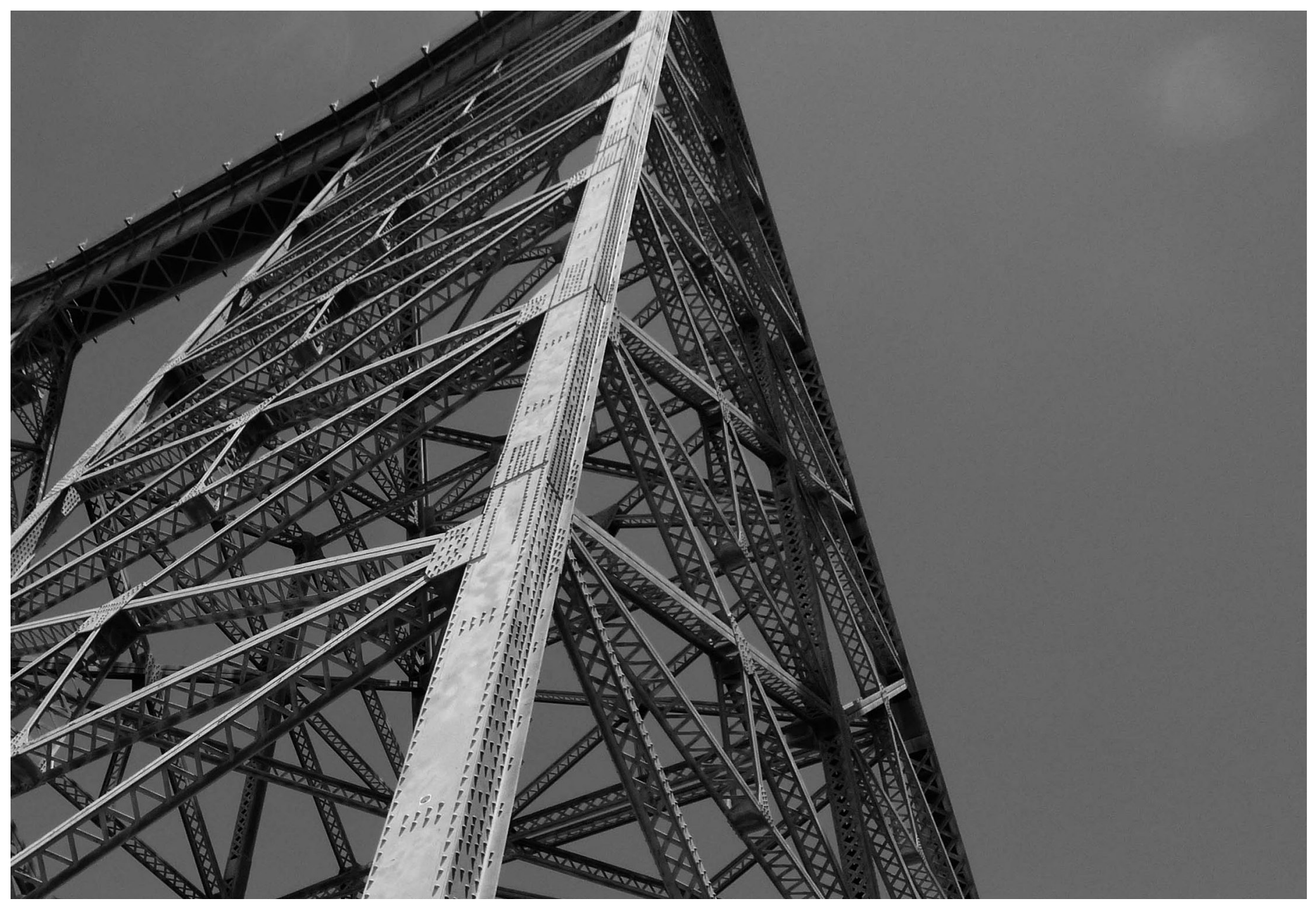

(c) Micaela Block 


\section{Logros y dificultades}

El primer acercamiento de la propuesta al jardín privado que depende de una universidad sirvió como plataforma para encarar el itinerario didáctico con avances, pero también con muchas dudas y planteos. Su aceptación significó trabajo, discusiones, reuniones docentes, entrevistas con padres, para determinar su implementación así como la resolución y carga horaria de las actividades y juegos.

La experiencia implicó transformaciones tanto en la comunidad de los jardines como en los alumnos universitarios y en la propia cátedra.

En los jardines, la aceptación y la respuesta por parte de los niños y familias acrecentaron el compromiso y la voluntad de los participantes del proyecto, por lo que se extendió la propuesta a toda la comunidad educativa universitaria. En este primer momento, donde solo participó una institución, los padres evaluaron y respondieron positivamente el proyecto. Algunas de las opiniones se presentan a continuación:

"Mi hijo, que concurre a la sala de 2 años en el turno tarde, reconoce muchas frutas y verduras que antes no nombraba. Las distingue no solo en la comida elaborada, sino también en plantas frutales. En huertas o espacios a los que a veces vamos de paseo cuando vamos a la verdulería las señala por su nombre con alegría. Puede diferenciar frutas de verduras y también distingue alimentos de otros grupos como los lácteos (leche, yogures, quesos) o huevos y también algo que me llamo la atención, es que destaca la comida que tiene 'semillas', como algunas tostadas o galletas. Algo más que creo que adquirió como un habito muy sano es la ingesta de de agua, pide en casa, en cualquier horario, o cada vez que salimos pone en mi bolso una botella con agua para tomar si tiene ganas. Veo que disfruta el momento de la comida, probando nuevas propuestas, eligiendo por su nombre también las carnes (pollo, pescadito, asado) y considero que es mérito del trabajo en conjunto con propuestas tan concretas y compartidas en el Jardín a través de la degustación y el juego". (Mariana R., mamá de Guido)

"Agostina reconoce perfectamente los vegetales y las frutas, y no solo a través de las imágenes, fotos o dibujos que puede ver en revistas o diferentes medios, sino también cuando las ve en la heladera, una frutera o una verdulería. Por otro lado, el reconocimiento de nuevas verduras despeja el temor asociado al mal gusto que podía llegar a tener hacia el sabor de algunas de ellas. Además, sabe distinguir entre las frutas cuáles pueden ser comidas con cáscara y cuáles no. Creemos que el proyecto aportó nuevos conocimientos e influyó positivamente en los niños". (Marcela N., mamá de Agostina)
Luego se sumaron los Jardines Municipales, cuya impronta y desarrollo de la PEEE fue diferente debido a las características de cada barrio y al trabajo de las docentes. Las actividades incluyeron conjuntamente a los niños de 1 a 3 años en un espacio lúdico común, por lo que los recorridos didácticos se programaron a conciencia y con la colaboración de todos los participantes del proyecto. Esto resultó enriquecedor en cuanto al trabajo interdisciplinario, la participación de la comunidad, la preparación de los recursos novedosos, prácticos, donde la bibliografía y material acorde al grupo etáreo sirvieron de base para ludotecas en las cátedras que participaban y en los propios jardines. Otra de las fortalezas fue que los niños acceden a la colación saludable que generalmente consta de frutas debido a su vinculación con instituciones que apadrinan a los jardines, así que la aceptación del proyecto por parte de la comunidad fue en su totalidad. Como dificultad, los talleres en los jardines, por otras actividades planificadas o por motivos extrainstitucionales, no se realizaron en la fecha programada y pasaron semanas sin que se efectuaran los encuentros con los niños.

Con respecto a los alumnos de la Universidad, estas prácticas de extensión fueron el nexo entre lo teórico con lo real y vivencial, se pudo vincular conceptos aportados por cátedras diferentes así como técnicas participativas sociocomunitarias adaptadas a los diferentes grupos. Reflexionar sobre lo aprendido y transferir a los niños a través de estrategias adecuadas fue un desafío permanente que retroalimentó el trabajo cotidiano.

También construyeron vivencias que reflejan el compromiso con lo social, así como actitudes y valoraciones en cuanto al niño y a las familias verificables en las devoluciones y autoevaluaciones presentadas a la cátedra. Para algunos, fue un modo de insertarse laboralmente en un campo de trabajo casi desconocido para el Licenciado en Nutrición.

Con referencia a la asignatura, se observó un crecimiento en el trabajo interdisciplinar, la vinculación con las instituciones y el medio, y además se generó el replanteo de contenidos así como la evaluación de la estructura del programa de estudios.

La evaluación se efectuó a lo largo de todo el proceso, con la participación de alumnos, docentes y directivos. La observación participante y el registro diario de las actividades realizadas con niños, familia y los docentes, fueron parte de la metodología utilizada. Los resultados consistieron, en general, en una valoración positiva. Sin embargo, demandaron la necesidad de vincular espacios curriculares con instituciones que agrupan a niños pequeños, donde sea posible modificar hábitos y estilos de vida saludable en edades tempranas. Además surgió la necesidad de replicar en otras instituciones educativas. 
Los futuros Licenciados en Nutrición, los docentes, padres y organismos gubernamentales tienen el gran desafío de bregar por la promoción de hábitos que permitan mejorar la calidad de vida de las personas.

A partir de esta propuesta, los estudiantes de la carrera en Nutrición se vinculan directamente con jardines de infantes de la ciudad y, en forma indirecta, con los acompañantes de los niños (familiares, tutores, maestras, etc.), puesto que transitan juntos una etapa considerada crítica en la formación de hábitos de salud y alimentarios nutricionales.

Esta propuesta, por un lado, impactó en los niños, ya que, a partir de juegos, estrategias didácticas y puestas en escena de una obra infantil que utiliza diferentes lenguajes artísticos expresivos con una impronta innovadora, divertida y colorida, pudieron incrementar su interés y agrado por el consumo de frutas, alimentos familiares, sanos y un estilo de vida activo. Por otra parte, para el alumno practicante y el equipo extensionista significó comprender que a través de la integración con la comunidad y metodología adecuada se favorece la adquisición de conceptos y procedimientos que posibilitan la comprensión y el desarrollo de la salud integral del niño. Además, el recorrido y aprendizaje vivencial implicaron resolver complejas tramas interpersonales y acompañar situaciones problemáticas con soluciones diversas e interdisciplinarias. La estructura del trabajo extensionista promovió la capacitación y trabajo en conjunto de los docentes del nivel inicial y futuros profesionales de la salud y nutrición, pero por sobre todo un contexto que facilitó la cohesión grupal al priorizar vínculos y las relaciones interpersonales.

En cuanto a la tarea docente, después del acompañamiento, orientación y colaboración en las diferentes etapas del equipo extensionista, se optimizó de tal manera que los alumnos pudieron intervenir activamente no solo en la diagramación de actividades y propuestas sino también en la transferencia de contenidos adecuados a los distintos grupos de trabajo y adaptando los mismos a las distintas realidades institucionales y socioeducativas (Jardines Municipales y jardín privado).

Las instancias y experiencias llevadas adelante deberían ser coordinadas con otros organismos oficiales, ministerios y universidad para beneficiar proyectos destinados a acompañar el desarrollo de los niños, pero se debe seguir insistiendo y comprometer, además, a los medios de comunicación para que el mensaje de un ambiente sano, así como una nutrición adecuada y armónica, llegue a toda la población.

Esto se enriquecerá permanentemente con los conocimientos específicos de cada área integrando las funciones sustantivas de la universidad: extensión, docencia e investigación. Numerosos convenios reflejan este propósito y plantean acciones futuras en conjunto con la Municipalidad y la Subsecretaría de Educación, Medio Ambiente, así como con los Ministerios de Educación, de Salud y de Desarrollo Social de la provincia de Santa Fe.

Es gratificante el pedido de proyectar la propuesta en los 13 Jardines Municipales de la ciudad y la socialización de los recursos construidos en diferentes organizaciones que atienden y bregan por el desarrollo de los niños del nivel inicial.

\section{Referencias bibliográficas}

Camilloni, A. (2011). Curso de Formación "La extensión como herramienta para la formación universitaria” (aprobado por Resolución decanal 047/11). Organizado en el marco del Programa de Mejoras de la Carrera de Arquitectura. FADU-UNL,

8, 17 y 18 de junio de 2011. FADU-UNL. Santa Fe, Argentina.

Del Campo, M. L.; Vara Messler, M. \& Navarro, A. (2010). Nutrition education in municipal primary schools in Cordoba: An experience in participatory action research (par) in 2008. Diaeta, 28 (132), 15-22. Recuperado el 27 de abril de 2016 de: http://www.scielo.org.ar/scielo.php?script=sci_arttext\&pi$d=S 1852-73372010000300003 \&$ lng=es\&tIng=en

Kain, J.; Leyton, B.; Concha, F.; Weisstaub, G.; Lobos, L.; Bustos, N. y Vio, F. (2012). Evaluación de una intervención en educación alimentaria y actividad física para prevenir obesidad infantil en escuelas públicas de Santiago de Chile. Archivos Latinoamericanos de Nutrición, 62 (1), 60.

Lifter, K. (2000). Linking Assessment to Intervention for Children with Developmental Disabilities or At-Risk for Developmental Delay: The Developmental Play Assessment DPA Instrument. En Gitlin-Weiner, K.; Sand Grund, A. y Schaefer, Ch., Play Diagnosis and Assessment ( $2^{\mathrm{a}}$ ed.). Nueva York: John Wiley\& Sons Inc. Ministerio de Educación, Argentina (2011). Proyecto de Alfabetización Científica; Food and Agriculture Organization FAO. Serie ciencia Salud y ciudadanía 2. Disponible en: http://repositorio.educacion.gov.ar:8080/dspace/handle/123456789/54487 Ortega Ruiz, R. (1992). El juego infantil y la construccion social del conocimiento. Sevilla: Alfar Universidad, 67. Serie Investigación y ensayo. 